\title{
Vasomotor reflex testing in leprosy patients, healthy contacts and controls: a cross-sectional study in Western Nepal
}

E. WILDER-SMITH, *A. WILDER-SMITH, $\dagger$ W. H. VAN BRAKEL \& †M. EGGER $\ddagger$

*Neurological Department, University of Berne, CH-3010 Switzerland, †Green Pastures Hospital, Pokhara, Nepal, $\neq$ Department for Social and Preventive Medicine, University of Berne, CH-3012 Switzerland

Accepted for publication 13 June 1996

Summary Objective: To examine test characteristics of laser Doppler vasomotor reflex testing for leprosy and to determine the prevalence of abnormal responses in leprosy patients, healthy contacts and controls.

Design and participants: Cross-sectional study including 89 leprosy patients (mean age 35 years, $74 \%$ male), 36 healthy contacts ( 29 years, $64 \%$ male) and 47 controls (30 years, $68 \%$ male), for a total of 172 participants.

Setting: Leprosy hospital in an endemic region $200 \mathrm{~km}$ west of Kathmandu, Nepal.

Outcome measure: Finger-tip and toe-tip vasomotor reflexes elicited by inspiratory gasp were measured using a laser-doppler flow temperature technique. Results were expressed in per cent as the maximal reduction in bloodflow from baseline.

Results: For all 12 measurement sites there were highly significant $(p>0.0001$ to $<0.004)$ differences between the three groups tested. Leprosy patients consistently had the lowest responses and controls the highest, with healthy contacts showing intermediate values. Thresholds defined as mean bloodflow reductions among controls minus 1.64 or minus 1.96 standard deviations provided optimal combinations of sensitivity and specificity. Using these cut-off values around $80 \%$ of leprosy patients, $50 \%$ of healthy contacts and $20 \%$ of controls had two or more abnormal reflexes ( $p<0.0001$ for differences between groups).

Conclusions: In endemic regions, subclinical autonomic neuropathy may be an early but detectable marker for the risk of subsequent leprosy, making early treatment and prevention of transmission possible. Prospective studies are needed to establish the predictive value of abnormal vasomotor reflexes.

\section{Introduction}

The prevention of disability in leprosy depends on the early detection and treatment of neural impairment. ${ }^{1}$ Treatment if implemented early prevents development of deformity, 
disability, results in a cure and eliminates transmission of further infection. However the early detection of leprous involvement of the peripheral nervous system is difficult, as even before clinical signs of leprosy are evident, there is evidence that extensive nerve damage has already taken place. ${ }^{2,3}$ As early detection of leprosy neuropathy is based on clinical sensory and motor testing, ${ }^{4,5}$ a considerable amount of underlying neural damage is present at the time of diagnosis. Much of the nerve damage will be irreversible. This and the recently proposed WHO goal of World-wide elimination of leprosy by the year $2000^{6}$ has fuelled the research for detecting the earliest possible paraclinical changes in leprosy neuropathy.

As there is good evidence that the focus of early infection is directed against ${ }^{2,7}$ autonomic nerve fibres, testing of autonomic nerve parameters is one possibility for early detection of leprosy. This is supported by the fact that several researchers have found impaired autonomic nerve reflexes in apparently healthy contacts of leprosy patientspossibly earliest form of leprosy neuropathy. ${ }^{8-10}$ The vasomotor reflex is an autonomic nerve reflex with an efferent pathway carried by small non-myelinated or poorly myelinated nerves thought to be particularly vulnerable to damage from the inflammatory infiltrate of leprosy neuropathy. Testing of the reflex was developed by Low et al. ${ }^{11}$ using laser Doppler velocimetry, to enable detection of neuropathic autonomic abnormalities. The transient fall in fingertip blood flow following inspiratory gasp is measured. The test has been studied extensively in clinical and experimental context and shown to be reproducible and reliable in the detection of abnormal autonomic reflexes. ${ }^{8,11}$

In light of the above arguments in favour of early autonomic nerve fibre involvement in leprosy we have investigated the frequency and magnitude of vasomotor disturbances across the clinical and chronological spectrum of leprosy ranging from early untreated to patients released from therapy. As clinically healthy contacts of leprosy patients are likely to include the earliest stages (subclinical) of leprosy we compared the leprosy and control groups with a group of healthy contacts.

The study was conducted in an area endemic for leprosy in Pokhara, Western Nepal.

\section{Patients and methods}

\section{PATIENTS}

The study was conducted at the Green Pastures Hospital, Pokhara, which is an International Nepal Fellowship (INF) hospital run in collaboration with the leprosy programme of His Majesty's Government of Nepal. Pokhara is located $200 \mathrm{~km}$ west of Kathmandu, and lies at the foot of the Annapurna mountain range.

Three groups of patients were recruited: 1, leprosy patients aged 10-55 years; 2, healthy contacts aged 10-55 years; and 3, controls aged 10-55 years. Only patients with a diagnosis confirmed by standard clinical criteria ${ }^{12}$ were included. Patients were further classified with respect to bacillary load into multibacillary (MB) and paucibacillary (PB). The Ridley-Jopling classification was also used (TT, tuberculoid leprosy; BT, borderline tuberculoid; BB, borderline borderline; BL, borderline lepromatous, and LL, lepromatous leprosy). Healthy contacts were recruited among relatives and friends of patients living in the same house as untreated leprosy patients for at least 1 year before treatment commenced (intense contact group) and among health care professionals (physiotherapists, nurses and occupational therapists) working in close contact with leprosy patients 
for at least 5 years (less intense contact group). Control participants were recruited among acquaintances of hospital personnel who did not work at the hospital or have friends or relatives with leprosy. Subjects with a history of diabetes, alcohol abuse or a diagnosis of polyneuropathy attributable to other causes were excluded from any of the three groups. The study protocol planned for frequency matching of healthy contacts and controls to leprosy cases for sex, and for age within 10-year-age bands.

All study participants were clinically examined for evidence of motor, sensory and autonomic impairment. This included a motor strength assessment of lower and upper extremities, and touch sensory testing with monofilaments as described previously ${ }^{13}$ as well as clinical testing for evidence of impaired sweating by looking for loss of suppleness and cracking of skin. Hand and foot callouses were registered in all subjects as either extensive or nonextensive. In leprosy patients the number of skin lesions, the number of enlarged or tender nerves and the number of body areas affected was assessed, and a complete treatment history was taken.

The project was accepted by both the ethical committee of the Leprosy Control Project, Nepal and the University of Bern, Switzerland.

\section{MEASUREMENT OF VASOMOTOR REFLEXES}

The vasomotor reflex is defined as the vasoconstrictor response to an autonomic stimulus ${ }^{14}$ and is usually measured with laser Doppler velocimetry of microvascular bloodflow. The pulp near the tip of the digit is the best site for measurement ${ }^{11}$ as this area possesses a wealth of arteriovenous anastomosis under strict autonomic control, containing only vasoconstrictor fibres, ${ }^{15}$ thereby allowing measurement of unmodified vasoconstrictor responses. Different stimuli can be used to elicit the skin vasomotor reflexes, (VMR) and responses are fairly unif orm. ${ }^{12}$ We chose the inspiratory gasp since in a small pilot study it gave the largest and most consistent relex response and was easiest of all the stimuli to conduct. Further details on the principles of laser Doppler velocimetry flux recordings are in the literature. ${ }^{11,16-20}$

We measured vasomotor reflexes of all 10 fingers and the big toes in order to obtain a complete as possible assessment of autonomic disturbances. Low et al,'s guidelines to measurement ${ }^{11}$ were used. We summarize the procedure. As vasomotor reflexes are best measured at maximal vasodilation of the digit pulp microvasculature, all subjects were encouraged to relax whilst seated. Finger vasomotor reflexes were examined with the forearm and hand at heart level. The vasomotor reflexes were measured with the leg and foot in a horizontal position. Ambient temperatures above $26.5^{\circ} \mathrm{C}$ (mean, $30.5^{\circ} \mathrm{C}$; maximum, $34.5^{\circ} \mathrm{C}$ ) made heating of the extremeties unnecessary.

Bloodflow was measured using a laser Doppler flow-temperature monitor (Moor Instruments, Model DRT4, Axminster, England) with machine settings of: bandwidth $10 \mathrm{KHz}$; time constant $1 \mathrm{~s}$. Gain control and zeroing were automatic. Double-sided adhesive disk secured attachment of the combined laser Doppler and temperature sensor probe to the skin, so ensuring optimal alignment of probe and skin surface. The procedure was explained in the patient's mother tongue. Persons were asked to practise to take the quickest and deepest breath you can and hold it for $10 \mathrm{~s}$.

Vasomotor reflex testing was not begun until the inspiratory gasp was satisfactorily mastered and a stable baseline bloodflow was recorded for each subject. Subsequently the inspiratory gasp was performed. The onset of each inspiratory gasp was marked with 
an event marker and the resultant maximal reduction in skin bloodflow recorded and expressed as a percentage of the resting skin bloodflow. Microvascular bloodflow (in flux) and skin temperature (in ${ }^{\circ} \mathrm{C}$ ) was recorded simultaneously and continuously and each examination printed on paper. In each subject the inspiratory gasp was repeated three times and the maximal response taken for recording.

In order to assess the repeatability of test results a subgroup of control participants was re-examined on a different occasion.

The test has previously been shown to be reproducible. ${ }^{11}$

SAMPLE SIZE AND STATISTICAL ANALYSIS.

The minimal sample size was calculated to detect a difference in the prevalence of abnormal vasomotor response between leprosy patients and contacts or controls of $30 \%$ at a significance level of 0.05 and with a power of $90 \%$ for a range of contact or control prevalences from $5 \%$ to $30 \%$. Results indicated that around 50 participants in each group were needed for this purpose.

Vasomotor reflex responses were analysed by calculating the maximum percent reduction in bloodflow for each of the 12 measurement sites. Mean reduction and standard deviation (sd) were calculated for each measurement site and participant group. In the case of absent responses, reductions were set to zero. For each finger and toe different threshold values separating abnormal from normal responses were calculated by subtracting $2.58,1.96,1.64,1.28,1.04$ and $0.25 \mathrm{sd}$ from the corresponding mean value obtained in controls. An overall abnormal test was defined as two or more measurements with reductions below this threshold value, as recommended by Low et al. ${ }^{11}$ A receiver operating characteristic (ROC) curve was constructed to illustrate the influence of different threshold values on test sensitivity and specificity. Different threshold values were used in analyses comparing the prevalence of abnormal tests between groups.

Statistical evaluation of differences in responses between groups used generalized linear models for analysis of variance of unbalanced data. Linear regression models were computed to identify factors associated with lower responses in multivariate analysis. $\chi$-square tests were used for the univariate comparison of categorical variables. Repeatability of test results in controls was assessed by comparing values in paired $t$-tests, by plotting the differences between the two measurements against the mean of the two measurements and by calculating the repeatability coefficient described by Bland \& Altman. ${ }^{21}$ This coefficient is defined as $1.96 \mathrm{sd}$ of the differences between measurements. Therefore, $95 \%$ of differences will be included in the value given by the repeatability coefficient.

Probability values were not adjusted for multiple comparisons. The statistical packages EPI-INFO Version 6 (Centers for Disease Control and Prevention, Atlanta, USA) and SAS Version 6.08 (SAS Inc., Cary, NC, USA) were used for data entry and statistical analysis.

\section{Results}

PATIENT CHARACTERISTICS

A greater number of leprosy patients but less healthy contacts and controls than 
anticipated were recruited. In the final analysis there were 89 leprosy patients, 36 contacts (24 intensive and 12 less intensive) and 47 controls, for a total of 172 participants. Frequency matching of contact and control groups for age and sex proved difficult and was only partly achieved. Mean age was $35 \cdot 0$ years (range 11-55) among patients, $29 \cdot 4$ years (11-54) among contacts and $30 \cdot 2$ years, (10-55) among controls ( $p=0.013$ by analysis of variance). Male participants dominated all groups (74\% male participants among leprosy patients, $64 \%$ among contacts and $68 \%$ among controls; $p=0.5$ by $\chi$-square test). Because of the age difference between the three groups, an association between age and VMR was statistically tested for and not found.

Prevalences of extensive callouses was similar in all three groups (18\% among leprosy patients, $11 \%$ among contacts, $15 \%$ among controls, $p=0.6$ by $\chi$-square test).

The characteristics of leprosy patients are shown in Table 1. Most patients with leprosy were diagnosed less than one year ago (47\%), mean interval to time of diagnosis being 46 months. BT was the most common type of leprosy (42\%).

\section{VASOMOTOR REFLEXES}

A complete set of 12 measurements could be performed in all except 4 participants (one patients who had undergone amputations of fingers and three controls because of technical reasons). Ten or 11 measurements were performed in these cases.

Levels of resting bloodflow were generally similar among controls and healthy contacts but lower among leprosy patients. Among controls and contacts mean values fluctuated around 250 flux for thumbs. 300 flux for all other fingers and 125 flux for toes. The corresponding figures for leprosy patients were 210, 250 and 105 flux respectively.

Table 1. Characteristics of leprosy patients

\begin{tabular}{lc}
\hline $\begin{array}{l}\text { Time since diagnosis } \\
\text { (mean in months) }\end{array}$ & $\begin{array}{c}\text { Percentage } \\
\text { Patients }\end{array}$ \\
\hline New & $10 \%$ \\
$1-6$ & $26 \%$ \\
$7-12$ & $11 \%$ \\
$13-24$ & $16 \%$ \\
$25-72$ & $15 \%$ \\
$73-396$ & $21 \%$ \\
& \\
Leprosy classification & \\
MB (multibacillary) & \\
PB (paucibacillary) & $50 \%$ \\
Ridley-Jopling & $50 \%$ \\
TT & \\
BT & \\
BB & $2 \%$ \\
BL & $42 \%$ \\
LL & $3 \%$ \\
PN & $29 \%$ \\
Release from therapy & $18 \%$ \\
& $6 \%$ \\
& \\
& \\
& \\
& \\
& \\
&
\end{tabular}


Table 2. Vasomotor reflex in leprosy, controls and contacts

\begin{tabular}{|c|c|c|c|c|}
\hline & Leprosy & Contacts & Controls & $p^{*}$ \\
\hline \multicolumn{5}{|l|}{ Right hand } \\
\hline Thumb & $42 \cdot 8(22 \cdot 9)$ & $53 \cdot 3(22 \cdot 0)$ & $62 \cdot 1(14 \cdot 1)$ & $0 \cdot 0001$ \\
\hline $\mathrm{R} 2$ & $51 \cdot 2(20 \cdot 2)$ & $59.6(20 \cdot 3)$ & $66 \cdot 8(15 \cdot 4)$ & 0.0001 \\
\hline $\mathrm{R} 3$ & $55 \cdot 3(21 \cdot 5)$ & $60 \cdot 3(24 \cdot 0)$ & $66.9(15.4)$ & 0.009 \\
\hline $\mathrm{R} 4$ & $51.8(20 \cdot 6)$ & $58 \cdot 8(22 \cdot 1)$ & $70 \cdot 8(12 \cdot 3)$ & 0.0001 \\
\hline R5 & $50 \cdot 2(23 \cdot 7)$ & $66.6(18.8)$ & $72 \cdot 3(17 \cdot 7)$ & 0.0001 \\
\hline \multicolumn{5}{|l|}{ Left Hand } \\
\hline Thumb & $44 \cdot 6(22 \cdot 5)$ & $55 \cdot 9(21 \cdot 2)$ & $63 \cdot 6(16 \cdot 3)$ & 0.0001 \\
\hline L2 & $47 \cdot 5(23 \cdot 1)$ & $56 \cdot 2(19 \cdot 7)$ & $68 \cdot 1(16 \cdot 6)$ & 0.0001 \\
\hline L3 & $51.8(23.0)$ & $58.9(23.9)$ & $69.9(14.0)$ & $0 \cdot 0001$ \\
\hline L4 & $53.8(22.8)$ & $63 \cdot 7(17 \cdot 3)$ & $72 \cdot 1(10 \cdot 5)$ & 0.0001 \\
\hline L5 & $50 \cdot 7(24 \cdot 1)$ & $66 \cdot 7(20 \cdot 8)$ & $72 \cdot 1(15 \cdot 3)$ & 0.0001 \\
\hline \multicolumn{5}{|l|}{ Feet } \\
\hline Left big toe & $41 \cdot 4(21 \cdot 6)$ & $56 \cdot 3(21 \cdot 7)$ & $60 \cdot 9(16 \cdot 4)$ & 0.0001 \\
\hline R. big toe & $41 \cdot 2(20 \cdot 9)$ & $55.6(19.8)$ & $56 \cdot 6(17 \cdot 4)$ & $0 \cdot 0001$ \\
\hline Combined & $48.9(15 \cdot 0)$ & $60 \cdot 1(14 \cdot 7)$ & $67.0 \quad(7 \cdot 6)$ & 0.0001 \\
\hline
\end{tabular}

${ }^{*} p$ is calculated by analysis of variance

The mean reduction of bloodflow with inspiratory gasp is expressed in percentage. Standard deviation is given in brackets.

R 1-5, right-handed digits 1-5; L 1-5, left-handed digits 1-5;

R6, right big toe; L6, left big toe.

Mean percent reduction of bloodflow for the different measurement sites and groups are shown in Table 2. Leprosy patients consistently had the lowest responses and controls the highest with healthy contacts showing intermediate values. The differences between the three groups were statistically highly significant $(p=0.0001$ in most instances). Significance levels were little affected when adjusted for age and sex. There were 12 patients $(13.5 \%)$, one contact $(2 \cdot 7 \%)$ and three controls $(6.4 \%)$ with one or more absent responses. In all groups responses were lower in big toes and thumbs than in the fingers. In controls, responses tended to increase from finger II to finger IV. No differences were noted between right and left sides.

Multivariate linear regression models showed that in leprosy patients lower responses were generally associated with positivity in skin smears and with a long history of disease. A detailed analysis of associations between reflexes and clinical characteristics will be presented elsewhere. In some regression models older age was weakly associated with decreased responses. In contacts and controls there was no association with age. Generally lower responses were recorded in the intensive contact group as compared to the less intensive group, although this did reach conventional levels of significance $(p=0.03)$ only for one measurement site. The results of the contact group will be presented in detail elsewhere.

The ROC curve (Figure 1) shows the relationship between different threshold values and test sensitivity and specificity. Table 3 gives test sensitivity, specificity and prevalence of test positivity for a range of threshold values. High specificity (but low sensitivity) is obtained with high threshold values defined as control mean minus 2.58 sd while a low threshold of control mean minus 1.28 sd optimized sensitivity at the cost of specificity. For all thresholds pronounced and statistically highly significant $(p<0.0001)$ differences 


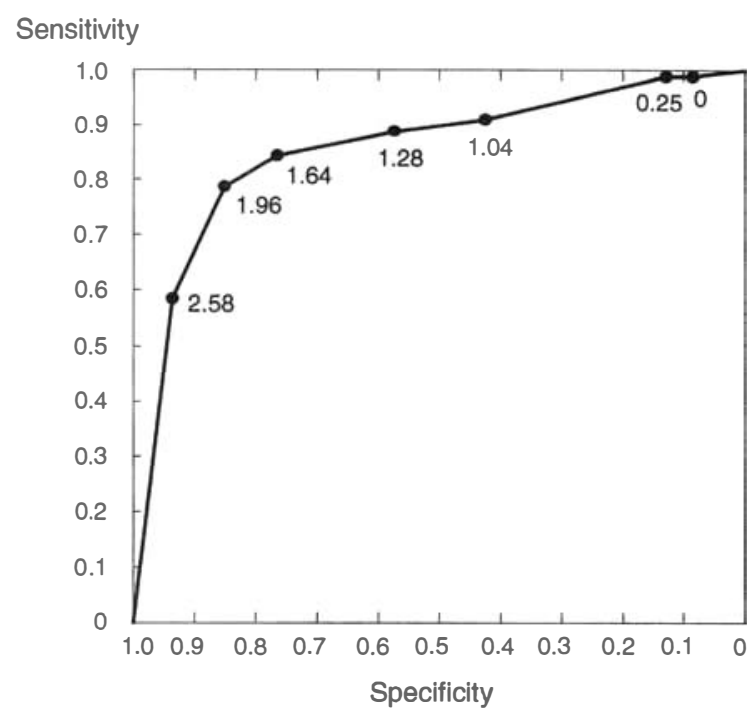

Figure 1. Receiver operating characteristic (ROC) curve for laser doppler vasomotor reflex testing in leprosy. The figures define cut-off points as the number of standard deviations subtracted from mean reductions in bloodflow.

in the prevalence of test positivity were evident between the three groups with the highest prevalence being evident among leprosy patients and the lowest among controls.

Comparing intense contact with less intense contact groups, generally higher prevelances were found among intense contacts, however these differences did not reach statistical significance $(p>0.03)$. The prevalence of test positivity among intense and less intense contacts was $25 \%$ and $25 \%$ (2.58 sd threshold), $54 \%$ and $42 \%$ (1.96 sd threshold), $58 \%$ and $50 \%$ (1.64 sd threshold), and $67 \%$ and $50 \%$ (1.28 sd threshold).

\section{REPEATABILITY}

Seventeen controls could be re-examined 3-21 days (mean 10 days) after the initial

Table 3. Test sensitivity, specificity and prevalence of abnormal test results according to the definition of threshold values

\begin{tabular}{|c|c|c|c|c|c|}
\hline \multirow{2}{*}{$\begin{array}{l}\text { Threshold } \\
\text { (No of sd minus mean) }\end{array}$} & \multirow{2}{*}{$\begin{array}{c}\text { Sensitivity/ } \\
\text { Specificity } \\
\%\end{array}$} & \multicolumn{4}{|c|}{ Prevalence of Test Positivity } \\
\hline & & Patients & Contacts & Controls & $\mathrm{P}^{*}$ \\
\hline $2 \cdot 58$ & 58.4 / 93.6 & $58 \cdot 4$ & $25 \cdot 0$ & $6 \cdot 4$ & $<0.0001$ \\
\hline 1.96 & $78 \cdot 7 / 85 \cdot 1$ & $78 \cdot 7$ & $50 \cdot 0$ & $14 \cdot 9$ & $<0.0001$ \\
\hline $1 \cdot 64$ & $84 \cdot 3 / 76 \cdot 6$ & $84 \cdot 3$ & $55 \cdot 6$ & $23 \cdot 4$ & $<0.0001$ \\
\hline $1 \cdot 28$ & $88.8 / 57 \cdot 5$ & $88 \cdot 8$ & $61 \cdot 1$ & $42 \cdot 6$ & $<0.0001$ \\
\hline
\end{tabular}

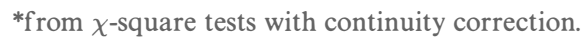
sd, standard deviation. 
examination. Mean absolute differences between the two measurements were between $-7 \%$ and $4 \%$ bloodflow reduction and probability values calculated from paired $t$-tests were between $0 \cdot 14$ and $0 \cdot 8$. There was thus no evidence of systematic error. However, plots of differences against the mean of the two measurements show considerable within person variability, with repeatability coefficients ranging from $23 \%$ to $47 \%$. A number of participants would have been classified differently after the second measurement. Taking the most stringent threshold $(2.58 \mathrm{sd})$ two out of three test positives were negative in repeat testing. Similarly, with the lowest threshold $(1 \cdot 28 \mathrm{sd})$ one out of three positives
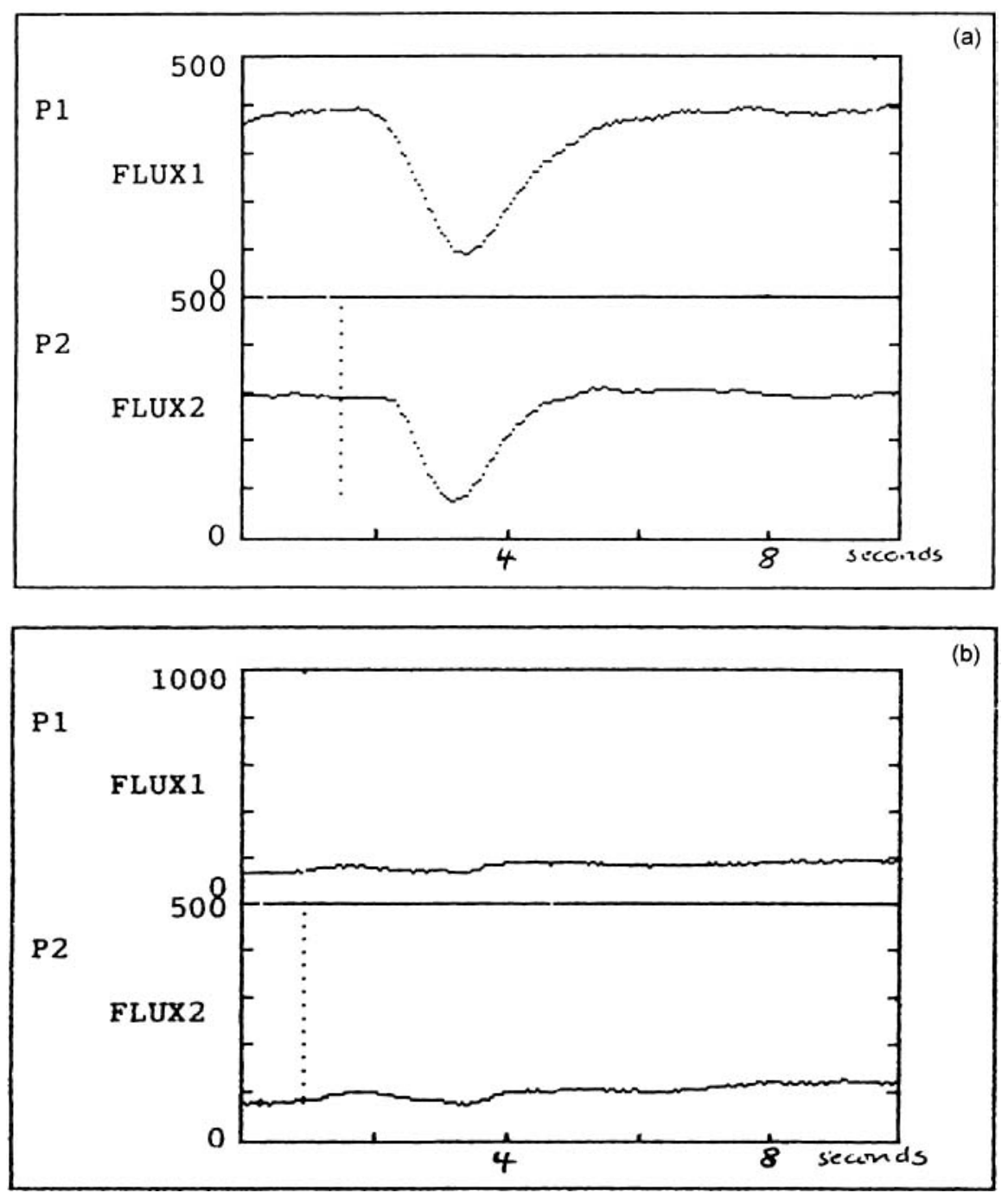

Figure 2. Examples of vasomotor reflexes. (a) Normal vasomotor reflexes in a Nepali control. P1, right third digit, P2, left third digit. The dotted line marks the inspiratory gasp. (b) Pathological vasomotor reflex in a leprosy patient. P1, right big toe, P2, left big toe. The dotted line marks the inspiratory gasp. 
were negative in the second test. No initially test negative participant was later found to be positive.

Figure 2(a) illustrates the normal vasomotor reflex of both third digits in a healthy Nepali control subject. The sharp and steep fall in fingertip blood flow by $75 \%$ can be seen to occur $2 \mathrm{~s}$ after inspiratory gasp. Five seconds later the bloodflow has returned to its previous level. Figure 2(b) shows the pathologically reduced vasomotor reflex of both big toes in a leprosy patients.

\section{Discussion}

Leprosy is endemic in subtropical and tropical regions with an estimated 11-15 million people suffering active disease worldwide ${ }^{22}$ with many more being infected. In approximately $5 \%$ of infections the disease leads to impairment due to blindness or deformities of the feet and hands. There is evidence that autonomic nerve damage in leprosy occurs before clinical manifestations become apparent. ${ }^{2,23}$ By the time clinical signs are present, extensive and often permanent nerve damage has taken place. A method able to detect asymptomatic neuropathy could be of value to identify patients at high risk for symptomatic leprosy. One possibility is the measurement of damage to autonomic nerves using vasomotor reflex testing. The aim of the present study was to examine characteristics of a laser Doppler vasomotor reflex test system and to investigate differences in test outcomes between leprosy patients, healthy contacts and controls.

Results indicate important differences between the three groups, however, groups could only incompletely be matched for age and sex. It proved particularly difficult to recruit healthy contacts, perhaps because of the social stigma associated with leprosy in Nepal. The male preponderance in the leprosy group may be explained by the higher incidence in males and possibly by better access of males to specialized care in a leprosy hospital. The age and sex differences between the three groups were taken into account in multivariate analyses with little effect on results. Prevalences of extensive callouses of the extremities was similar across the groups.

Among controls resting bloodflow was higher and reflexes were more pronounced than reported previously. ${ }^{11}$ This may be explained by the constant high ambient temperatures and high levels of humidity favouring maximal skin vasodilation and thus creating optimal testing conditions. For all 12 measurement sites there were pronounced and statistically highly significant differences in responses between the three groups. The strongest responses were consistently recorded in control participants and the least among leprosy patients, with healthy contacts in an intermediate position. Test characteristics and the prevelance of overall abnormal tests depend on the definition of normality. This is illustrated by the ROC curve which indicates that a cut-off value defined as the mean reduction in bloodflow among controls minus 1.64 to $1.96 \mathrm{sd}$ provides optimal combination of sensitivity and specificity. Using these cut-off values around $80 \%$ of leprosy patients, $50 \%-55 \%$ of healthy contacts and $15 \%-23 \%$ of controls have abnormal results.

The high rate of abnormal tests a mong controls is unexpected. There are two factors that may contribute to this. First, in the area of recruitment there is a concentration of leprosy patients from the hospital (100 inpatients, 4000 outpatients visits per year). A number of control participants could in fact have been exposed and should correctly be 
classified as healthy contacts. They may have been reluctant to acknowledge contact to leprosy patients or may not have been aware of such contact. Other causes of abnormal VMR such as other polyneuropathies are unlikely since diabetes and a history of alcohol abuse were excluded.

Second, when re-examining a subgroup of controls a proportion of control participants with abnormal tests were normal on repeat testing, perhaps because of lack of compliance of the examinee during the first round of testing. Based on the literature, ${ }^{11}$ we assumed adequate reliability of the method and therefore did not perform a prior reliability investigation. However, the current results indicate that reliability may have been less good than expected. We believe that this does not invalidate the main results, but further confirmation of this will be necessary. In particular, a thorough reliability study of laser Doppler flowmetry will need to be done.

We do not think it likely that subjects with previously successfully treated leprosy were included since distribution of antilepromatous therapy is government controlled and all cases of leprosy are registered and controlled. In addition the leprosy hospital has excellent treatment records of all leprosy patients treated over the past 40 years and documentation of contact examination. Contacts were all well known to the hospital staff. In the setting of this selected community, hidden leprosy treatment would have been evident.

The most important finding of our study is the high prevalence of subclinical neuropathy among healthy contacts. Within this group the prevalence tended to be higher among participants with intense contact and lower among those with less intense exposure. These findings confirm those from an earlier study conducted in India and Scotland. ${ }^{9}$ The data strongly support the notion that autonomic nerve damage represents an early manifestation of the disease process. Indeed histopathological and immunocytochemical studies have demonstrated that nerve damage progresses from small unmyelinated to small myelinated and finally to large myelinated fibres. ${ }^{23,24}$

Early treatment of leprosy prevents progression to deformity and disability and eliminates transmission of the disease. Treatment is well tolerated, cheap and available worldwide. ${ }^{25}$ Early detection of leprosy may thus be an important strategy which could substantially contribute to achieving the declared goal of worldwide elimination of leprosy. ${ }^{6}$

Screening for asymptomatic disease using vasomotor reflex testing could play an important role in this effort. A number of obstacles, however, need to be overcome before such screening could sensibly be implemented. First and an important principle of early disease detection, the progression from asymptomatic early stages to clinical disease needs to be understood more completely. ${ }^{26}$ In leprosy, it is not clear at present if neuropathy detected among healthy contacts does in fact always represent an early stage of an active disease process. Alternatively, it could correspond to an 'immunopathological scar' associated with an earlier infection which was successfully overcome by the immune system. A parallel may be drawn to another mycobacterial infection, tuberculosis. After exposure to Mycobacterium tuberculosis the immune response results in the formation of a Ghon complex. In many cases the disease will not progress further as there is adequate immunity. Activation may nevertheless occur at a later time. Similarly, after exposure to $M$. leprae, the immune response may result in a 'Ghon-complex-type' damage to the peripheral autonomic nerves. Progression to symptomatic disease may or may not occur depending on the course of the interaction between host and bacteria. The 
risk of progression to clinical disease among different groups of people with abnormal vasomotor responses clearly needs to be established in prospective studies before recommendations can be made regarding screening for and treatment of asymptomatic disease. Sampatavanich et al. ${ }^{27}$ performed an immunoepidemiological study on subclinical infection among leprosy household contacts in Thailand and found that $33 \cdot 5 \%$ of contacts were inf ected and probably a t risk to develop leprosy as they had no evidence of cell-mediated immunity against $M$. leprae. A study using lymphocyte transformation tests found that people in contact with leprosy patients for more than one year had evidence of subclinical infection in more than $50 \%{ }^{28}$ Unfortunately the risk for developing symptomatic disease was not prospectively assessed in these studies.

Second, a rapid and simple test appropriate for mass screening and acceptable to the target population would have to be developed. The system used in this study is expensive and testing is still time consuming. This system could prove useful in tertiary centres to monitor selected patients for early progression of neuropathy. For example, titration of anti-inflammatory treatment during acute reactions would thus become possible. Finally, any screening programme would have to be evaluated in carefully designed studies, and, once implemented on a large scale, would have to continue for a sufficiently long period of time.

In conclusion, we have shown a strong association between autonomic neuropathy as assessed by vasomotor reflex testing and leprosy, both among those with manif est disease and among healthy contacts at high risk of infection. Carefully planned prospective studies are now needed to better define the significance of these findings for early treatment and prevention of transmission.

\section{Acknowledgments}

We are grateful to the International Nepal Fellowship and especially to Dr Frauke Wörpel, Superintendend of Green Pastures Hospital, and Dr Allison Anderson of the Leprosy Control Project Pokhara, to enable us to perform this study in Pokhara, West Nepal. We also want to thank Mr Khadga, Adga and Krishna of the Physiotherapy Department and $\mathrm{Mr}$ Khawas of the Leprosy Control Project for their valuable practicable help. The study was financially supported by the Swiss Academy of Medical Sciences and by Sandoz (Sandoz-Stiftung zur Förderung der Medizinisch-Biologischen Wissenschaften). M.E. was supported by the Swiss National Research Fund. Last but not least, this study would not have been possible without the help of many leprosy patients who were willing to partake in this study.

\section{References}

${ }^{1}$ Brakel WH. van Peripheral neuropathy in leprosy: the continuing challenge. Thesis; University of Utrecht, Holland, 1994.

2 Shetty VP, Mehta NH, Antia NH, Irani PF. Teased fibre study of early nerve lesions in leprosy and contacts, with electrophysiological correlates. J Neurol Neurosurg Psychiat 1977; 40: 708-711.

3 Shetty VP, Antia NH, Jacobs JM. The pathology of early leprous neuropathy. J Neurol Sciences 1988; 88: $115-131$.

${ }^{4}$ Palande DD, Bowden REM. Early detection of damage to nerves in leprosy. Lepr Rev 1992; 63: 60-72. 
${ }^{5}$ Fritschi EP. Field detection of early neuritis in leprosy. Lepr Rev 1987; 58: 173-177.

${ }^{6}$ Bechelli LM. Prospects of global elimination of leprosy as a public health problem by the year 2000. Int J Lepr 1994; 62: 284-292.

7 Karanth SS, Springall DR, Lucas S, Levy D, Ashby P, Levene MM, Polak JM. Changes in nerves and neuropeptides in skin from 100 leprosy patients investigated by immunocytochemistry. J Pathol 1989; 157: 15-26.

${ }^{8}$ Swanson Beck J, Abbot NC, Samson PD, Butlin CR, Grange JM, Cree IA, Forster A, Khan F. Impairment of vasomotor reflexes in the fingertips of leprosy patients. J Neurol Neurosurg Psychiat 1991; 54: 965-971.

9 Abbot NC, Swanson Beck J, Samson PD, Butlin CR, Brown AR, Forster A, Grange JM, Cree IA. Impairment of fingertip vasomotor reflexes in leprosy patients and apparently healthy contacts. Int J Lepr 1991; 59: 537-547.

10 Abbot NC, Swanson Beck J, Mobayen HM, Samson PD, Stanford JL. Reduced fingertip blood flow and peripheral dysautonomia in leprosy patients in Iranian Azerbaijan and in Maharashtra, India. In J Lepr 1993; 61: 474-476.

11 Low PA, Neumann C, Dyck PJ, Fealey RD, Tuck RR. Evaluation of skin vasomotor reflexes by using laser Doppler velocimetry. Mayo Clin Proc 1983; 58: 583-592.

12 Yawalkar SJ. Leprosy 5th ed., Ciba-Geigy Ltd Basle, Switzerland, 1992:64 ff.

13 Bell-Krotoski JA. Light touch-deep pressure testing using Semmes-Weinstein monofilaments. In: Rehabilitation of the hand, 3rd ed. Hunter et al. (eds) C.V. Mosby Co., 1989; 585-93.

14 Low PA. Autonomic nervous system function. J Clin Neurophysiol 1993; 10: 14-27.

15 Roddie IC, Sheperd JT, Whelan RF. A comparison of the heat elimination from the normal and nerve blocked finger during body heating. J Physiol (Lond) 1957; 138: 445-448.

16 Bonner RF, Clem TR. Laser-Doppler continuous real-time monitor of pulsatile and mean blood flow in tissue microcirculation. In: Chen SH, Chu B, Nossal R, eds. Scattering techniques applied to supramolecular and non-equilibrium systems. New York, Plenum Press 1981: 685-701.

17 Watkins D, Holloway GA. An instrument to measure cutaneous blood flow using the Doppler shift of laser light. IEEE Trans Biomed Eng 1978; 25: 28-33.

18 Nilsson GE, Tenland T, Åke Öberg P. Evaluation of a laser Doppler flowmeter for measurement of tissue blood flow. IEEE Trans Biomed Eng 1980; 27: 597-604.

19 Stern MD, Laappe DL, Bowden PD, Chimosky JE, Holloway GA Jr Keiser HR, Bowman RL. Continuous measurement of tissue blood flow by laser-doppler spectroscopy. Am J Physiol 1977; 232: H441-H448.

20 Oberg PA, Nilsson GE, Tenland T, Holmström A, Lewis DH. Use of a new laser doppler flowmeter for measurement of capillary blood flow in skeletal muscle after bullet wounding. Acta Chir Scand (Suppl) 1979; 489: 145-150.

${ }^{21}$ Bland JM, Altman DG. Statistical methods for assessing agreement between two methods of clinical measurement. Lancet 1986; i: 307-310.

22 Bell DR. Lecture notes on tropical medicine. 4th edition, Oxford:Blackwell Science, 1995:293.

23 Shetty VP, Mehta LN, Irani PF, Antia NH. Study of evolution of nerve damage in leprosy. Part 1-Lesions of the index branch of the radial cutaneous nerve in early leprosy. Part 2-Observation on the index branch of the radial cutaneous nerve in contacts of leprosy. Lepr India 1980; 52: 5-25.

24 Shetty VP, Mehta LN, Antia NH. Unmyelinated fibres in leprosy neuritis. An ultrastructural study. Bull Electron Microsc Soc India 1978; 2: 2-5.

25 Meyers WM, Marty AM. Current concepts in the pathogenesis of Leprosy. Drugs. 1991; 41: 832-856.

26 Wilson JMG, Junger G. Principles and practice of screening for disease. Geneva: World Health Organisation, 1969.

27 Sampatavanich S, Samponnachot P, Kongsuebhart K, et al. Immunoepidemiological studies on subclinical inf ection among leprosy household contacts in Thailand. Int. J. Lepr 1989; 57: 752-765.

${ }^{28}$ Godal T, Negassi K. Subclinical infection in leprosy. Brit Med J 1973; 3: 557-559. 